\title{
Theoretical studies on the calculation of the noise of impact equipment in blacksmith shops
}

\author{
Elena Staseva $^{1}$, Dmitry Larin ${ }^{1}$, Sergei Demchenko ${ }^{1}$, Kirill Kobzev ${ }^{1, *}$ \\ ${ }^{1}$ Don State Technical University, 1, pl. Gagarina, 344002, Rostov-on-Don, Russia
}

\begin{abstract}
Most types of forging equipment relate to impact machines, during the operation of which impulse noise occurs, the levels of which at workplaces, in most cases, exceed the maximum permissible values and relate to those factors, the significance of which in some cases is greater than dustiness, high temperature, manipulation of heated workpieces. Therefore, the problem of reducing vibration and noise in the forge shops is extremely relevant and has great scientific and socio-economic significance. To solve the problem of noise reduction in forge shops, various options for reducing the noise activity of equipment have been developed/ It is possible to most effectively reduce noise by changing the material and shapes of the beds, noise-attenuating casings, oil baths for gears.
\end{abstract}

\section{Introduction}

All these measures on existing and running equipment are implemented through the use of passive noise protection measures, which in the current production are little feasible, and in most cases impossible at all. The problem of reducing noise in the source of its occurrence and bringing the noise levels to the maximum permissible values should be solved at the design stage. Such an approach to solving the problem of noise reduction is the most justified both from the scientific, technical and economic points of view. Work in this direction is carried out in far abroad countries and is almost completely absent in our country. This is explained, first of all, by the absence of reliable engineering methods for calculating noise and vibration, which would take into account the design features of forging machines and technological operating modes, made it possible to assess the expected noise levels at the stage of calculation and design and choose engineering solutions to reduce noise levels up to standard values for each specific type of forging equipment.

When working in conditions of vibration, labor productivity decreases, the number of injuries increases. At some workplaces in agricultural production, vibrations exceed normalized values, and in some cases they are close to limit. The vibration levels on the controls do not always comply with the standards. Typically, low-frequency vibrations that negatively affect the body predominate in the vibration spectrum. Some types of vibration adversely affect the nervous and cardiovascular systems, the vestibular apparatus. The most

*Corresponding author: 5976765@mail.ru 
harmful effect on the human body is provided by vibration, the frequency of which coincides with the frequency of natural vibrations of individual organs, the approximate values of which are as follows $(\mathrm{Hz})$ : stomach - $2 \ldots 3$; kidneys - $6 \ldots 8$; heart - 4 .. 6; intestines - 2 ... 4; vestibular apparatus - 0.5..L, 3; eyes - 40 .. 100, etc.

The effect on muscular reflexes reaches $20 \mathrm{~Hz}$; the tractor-loaded seat on the tractor has its own vibration frequency of $1.5 \ldots 1.8 \mathrm{~Hz}$, and the rear wheels of the tractor - $4 \mathrm{~Hz}$. The vibration is transmitted to the human body at the moment of contact with the vibrating object: when acting on the limbs, local vibration occurs, and on the whole body - general. Local vibration affects neuromuscular tissues and the musculoskeletal system and leads to spasms of peripheral vessels. With prolonged and intense vibrations, in some cases, a professional pathology develops (local vibration often leads to it): peripheral, cerebral or cerebral-peripheral vibrational disease. In the latter case, there are changes in cardiac activity, general excitement, or, conversely, inhibition, fatigue, the appearance of pain, a feeling of shaking of the internal organs, nausea. In these cases, vibrations also affect the osteoarticular apparatus, muscles, peripheral circulation, vision, hearing $[1,3,5,7,9]$. Local vibrations cause spasms of blood vessels that develop from the terminal phalanges of the fingers, spreading to the entire hand, forearm, and cover the vessels of the heart.

Workers can be exposed to occupational vibration through the use of power or pneumatic hand tools or other machinery, or by driving large transportation, construction or agricultural vehicles. Vibration that is generated through the use of powered hand tools, and is transmitted from the tool to the hand-arm system is referred to as hand-transmitted vibration. However, recent studies have also demonstrated that vibration can be transmitted through platforms workers are standing on, and in these situations, the point of contact is the feet. Workers can also be exposed to whole body vibration (WBV). WBV exposure occurs in occupations where workers are driving trucks, large earth moving vehicles, or where they are using hand tools where the amplitude of the vibration is great enough to be transmitted to other portions of the body, such as in workers using jack-hammers.

Exposure to WBV is of concern within the workforce because it's associated with the development of a number of negative health consequences including back and neck pain, and potentially, cardiovascular disease, the development of various neuropathies, digestive problems, headaches, dizziness, motion sickness and possibly cancer. However, workers exposed to WBV are often also exposed to a number of other risk factors that may contribute to the development of these negative health effects. These risk factors include maintaining a static posture for a long-period of time, torque or twisting of the abdomen to view the area around the vehicle, and heavy lifting that often occurs when a vehicle is being loaded or unloaded. In addition to vibration and the physical exposures associated with a job, there may be other co-exposures to chemicals or certain environmental conditions that contribute to the development of disease or injury in workers. Because most workers are exposed to multiple factors that may induced injury or illness, it's difficult to determine which factors pose the greatest risk for inducing injury or illness. Experimental studies, examining the effects of each of these factor individually on health outcomes can provide additional information that will help determine the contribution of each exposure factor to various health problems.

This review will describe the industrial sectors where vibration exposure is most prevalent and the health effects associated with exposure to HTV and WBV. Experimental methods used to measure and characterize vibration generated in various occupational settings are discussed, and models that have been used to uncover the etiology of vibrationinduced injuries. Although numerous studies have been published on both HTV and HBV, there are new epidemiological studies showing an increased risk of specific cancers with exposure to WBV. Therefore additional etiological studies need to be performed. New avenues for research are discussed below. 


\section{Methods}

Noise reduction is achieved by creating new designs of low-noise automatic devices, for example, placing gears in oil baths, closing them with soundproof casings, replacing the power spring of the short circuit in the cam mechanisms of the drive slider by springs with kinematic closing and cams designed according to the law motion, providing a sinusoidal symmetric law of change of the accelerator of the pusher. These measures reduced noise by 5-9 dB [1,3,5,7,9].

A technique has been developed to calculate the sound pressure level of the machine during its design, based on the vibrational velocities of the surfaces of the structure. When calculating the sound pressure level, the equations of motion of the mechanisms are represented by the equation of oscillation in an exponential form without taking into account the resistance, while the amplitude of the vibration (noise) is proportional to the square of the angular velocity of rotation of the shaft and depends on the mass of the mechanism and the rigidity of its fastening. The equation of motion is represented as

$$
m \omega^{2} y_{a}+c y_{a}=p_{a} y=y e^{i \omega x}
$$

vibration speed

$$
\left[V_{0}\right]=y_{a} \omega=\frac{p_{a}}{\frac{c}{\omega}+\frac{\omega}{m}}
$$

Where $\mathrm{p}_{\mathrm{a}}$ - the amplitude of the disturbing forces, $\mathrm{s}$ and $\omega$ are the reduced stiffness and mass.

The authors determine the sound pressure level of the corresponding mechanism by the formula

$$
L=20 \lg \frac{V}{V_{0}}
$$

and the automaton as a whole according to the well-known formula of energy summation. The authors determine the safety factor by the sound pressure level of the machine [11]. The results of the work make it possible to calculate some mechanisms and designs of the machine, varying their rigidity and other parameters, which leads to a decrease in impact force and an increase in the accuracy of the machine setup. The stiffness of the supporting structure of the connecting rod and other parts is calculated based on sound pressure levels corresponding to hygiene standards. As a result, the oscillation speed of the corresponding mechanism is determined. For the period 0 -t 2 of application of the perturbing force $\mathrm{P}(\mathrm{f})$, the authors set the equation of motion of the wall of the supporting structure or the construction of the connecting rod in the form

$$
y=\frac{1}{\sqrt{2\left(m+\frac{m_{1}}{3}\right) \omega_{o}^{2}}} \int p(f) \sin \omega_{o}\left(t_{2}-t_{1}\right) d t
$$


Integrating it in parts and believing that, $\left(m+\frac{m_{1}}{3}\right) \omega_{o}^{2}=c$, the authors obtained an expression of the vibrational velocity at $P(f)=\frac{p(f)}{t_{2}}$

$$
\begin{gathered}
V=\frac{p(f)}{\sqrt{2} C t_{2}}-\frac{p(f)}{\sqrt{2} C t_{2}} \cos \omega_{o} t \\
L=20 \lg \frac{V}{V} \text { reduced to } L=20 \lg A \frac{1}{C t_{2}}
\end{gathered}
$$

where $\mathrm{A}$ is a value depending on the shape of the structure and its loading. The sound pressure level of covers, housings and other attached structures is calculated based on their soundproofing ability. In this case, the stiffness of the fences does not play a role and the growth of sound insulation is determined by the law of masses

$$
R=13,5 \lg G+13
$$

According to the authors, the application of the calculation method in the design of automatic machines makes it possible to create machines with characteristics that ensure compliance with hygiene standards in the workplace.

\section{Results}

The applicability of the given dependences for practical noise calculations at the design stage is quite limited. First of all, it should be noted that expression (3) describes the motion of a system with a concentrated mass, which makes it possible to determine the vibrational velocities of the mechanism at the natural vibration frequency, that is, it is applicable to the tonal spectrum of vibrations and noise $[2,4,6,8]$.

A similar technique is not applicable for the calculation of noise levels, which has a broad-band nature, which is typical for forging equipment. Expression (4) determines the level of vibration, and not noise, which is proportional to the vibrational speed.

The proposed one also allows you to determine the sound level (dBA), and not the levels of the spectral components of the noise spectrum

$$
L=(128)+\lg A-\lg M_{1}
$$

where: A - impact energy; $\mathrm{M}$ - mass of falling parts.

Intense noise emission is created by the frame and flywheel of a mechanical press. The vibrations of the bed and the flywheel are excited as a result of impacts in all movable joints of the press that occur at the moment of switching on and at the beginning of the movement of the crank or eccentric mechanism, that is, when the gaps in the joints of the connecting rod with the working shaft neck and slider are sampled bearings of the working shaft. The process of interaction between the stamp and the workpiece also has an impact character [10]. The increase in stamping noise levels reaches $10 \mathrm{dBA}$. There is no noise of turning on the press during automatic operation. Noise levels do not change compared to the single start mode. Noise reduction can be achieved by ensuring a smooth operation of 
the switching process, which is realized by replacing mechanical cam couplings with friction or pneumatic ones. Noise during stamping is reduced when using beveled dies, instead of straight ones, which contributes to a smooth working process [12]. These measures allow to reduce noise at the workplace of the puncher by $14 \mathrm{dBA}$. The use of such dies is justified when cutting parts of a large perimeter.

Gaskets are plates made of hard rubber, rubber-fabric material, asbestos-rubber, perforated neoprene, hard cardboard, pagulan, volculon materials from 1 to $6 \mathrm{~mm}$ thick, placed at the joints of the bed stands with the scabbard, and the racks with a sub-cylinder a stove and a cylinder of hammers and between wedges and racks. Elastic films at the same joints are made by applying gumming nairite composition or SKU-PFL polyurethane hammer in several layers with a total thickness of $1 \mathrm{~mm}$ to the primed contact surfaces of the parts. Measures to separate the butt surfaces and tighten the joints, together with the connection of the uprights in the upper section with tie bolts with an elastic element, reduce noise by 4-10 dBA. Rigid wedge fastening of the sub-cylinder plate to the uprights by the connection "lasochtokin tail" is also effective.

Intense noise emission is created by the frame and flywheel of a mechanical press. The vibrations of the bed and the flywheel are excited as a result of impacts in all movable joints of the press that occur at the moment of switching on and at the beginning of the movement of the crank or eccentric mechanism, that is, when the gaps in the joints of the connecting rod with the working shaft neck and slider are sampled bearings of the working shaft. The process of interaction between the stamp and the workpiece also has an impact character. The increase in stamping noise levels reaches $10 \mathrm{dBA}$. There is no noise of turning on the press during automatic operation. Noise levels do not change compared to the single start mode. Noise reduction can be achieved by ensuring a smooth operation of the switching process, which is realized by replacing mechanical cam couplings with friction or pneumatic ones. Noise during stamping is reduced when using beveled dies, instead of straight ones, which contributes to a smooth working process. These measures allow to reduce noise at the workplace of the puncher by $14 \mathrm{dBA}$. The use of such dies is justified when cutting parts of a large perimeter.

Preliminary operations (rolling, broaching, draft) of stamping on hammers are advisable to perform on separate equipment (forging rollers, hammers), since when performing preliminary and final stamping on a single hammer, strikes with different energies are applied, which requires a highly skilled blacksmith when controlling a hammer. The use of software control of the energy of the hammer strike eliminates the possibility of a collision of the mirror stamps during the preliminary operations.

Studies have established that stamp vibrations are transmitted through the die holder and rammers to the racks and the hammer cylinder and, in addition, during eccentric impacts through the head and guides, also to the hammer racks.

To reduce the vibrational excitation of the hammer parts, between the shabot and the die holder, as well as between the wedge and the die holder, a $2 \mathrm{~mm}$ thick rubber-asbestos gasket is installed, withstanding pressure up to $200 \mathrm{kgf} / \mathrm{mm} 2$ and temperature up to $500^{\circ}$ $\mathrm{C}$, or made of rubber-like material of the FKS brand, placed in order to avoid extrusion from the joint into the recess on the supporting surface of the die holder. At the same time, the noise level is $4 \mathrm{dBA}$.

It is possible to prevent the propagation of vibration from the hammer's hammer to the bed by installing damping gaskets at the joints or by coating the contact surfaces with elastic films, as well as by installing more powerful polyurethane shock absorbers and by increasing the tightening forces of the joints, preventing them from opening and impacting the contact surfaces of the parts during stamping.

Gaskets are plates made of hard rubber, rubber-fabric material, asbestos-rubber, perforated neoprene, hard cardboard, pagulan, volculon materials from 1 to $6 \mathrm{~mm}$ thick, 
placed at the joints of the bed stands with the scabbard, and the racks with a sub-cylinder a stove and a cylinder of hammers and between wedges and racks. Elastic films at the same joints are made by applying gumming nairite composition or SKU-PFL polyurethane hammer in several layers with a total thickness of $1 \mathrm{~mm}$ to the primed contact surfaces of the parts. Measures to separate the butt surfaces and tighten the joints, together with the connection of the uprights in the upper section with tie bolts with an elastic element, reduce noise by 4-10 dBA. Rigid wedge fastening of the sub-cylinder plate to the uprights by the connection "lasochtokin tail" is also effective.

\section{Discussion}

The noise level on a hammer with a separate bed with an eccentric shock is 10-15 dBA higher than with a central one, which is associated with the excitation of vibration of the racks by the beam through the guides. To exclude eccentric impacts, preliminary stamping operations must be performed on special equipment (for example, forging rollers), and gaskets made of pagulan type material with a thickness of $1 \mathrm{~mm}$, designed for pressure up to 10 , should be installed in the joints of guides with racks of the bed of the hammer. kgf / $\mathrm{mm} 2$ and temperature up to $80 \mathrm{C}$.

It is shown that the noise level from a hammer with a round bed profile and a structural damper built into the cavity of the bed stands is $10 \mathrm{dBA}$ lower than from a hammer with an open bed profile. The profile of the Shabot must be convex in order to avoid the formation of a reflected wave.

The structural damping of the bed can be increased by closing the rack profile by attaching screens with a sound-absorbing coating (for example, from a layer of mineral wool) on it and installing partitions inside the closed cavities with filling the cells between the partitions with sand, gravel or shot.

The noise level on a hammer with a separate bed with an eccentric shock is 10$\urcorner-15 \mathrm{dBA}$ higher than with a central one, which is associated with the excitation of vibration of the racks by the beam through the guides. To exclude eccentric impacts, preliminary stamping operations must be performed on special equipment (for example, forging rollers), and gaskets made of pagulan type material with a thickness of $1 \mathrm{~mm}$, designed for pressure up to 10 , should be installed in the joints of guides with racks of the bed of the hammer. kgf / $\mathrm{mm} 2$ and temperature up to $80 \mathrm{C}$.

It is shown that the noise level from a hammer with a round bed profile and a structural damper built into the cavity of the bed stands is $10 \mathrm{dBA}$ lower than from a hammer with an open bed profile. The profile of the Shabot must be convex in order to avoid the formation of a reflected wave.

The structural damping of the bed can be increased by closing the rack profile by attaching screens with a sound-absorbing coating (for example, from a layer of mineral wool) on it and installing partitions inside the closed cavities with filling the cells between the partitions with sand, gravel or shot.

To combat noise and vibration, both general and individual protective equipment is used. When planning production facilities, such as an engine test station, thermal and blacksmith shops are located on the leeward side in relation to other buildings and a residential area.

To attenuate the noise penetrating outward, it is necessary to use sound insulation of building envelopes. The rationalization of technological processes, the use of silencers, the careful fit of all moving parts of mechanisms - all this many times reduces noise. The greatest effect is achieved by replacing noisy jobs with less noisy ones.

Pneumatic riveting of frames and other parts must be replaced by hydraulic riveting or welding, forging and stamping by pressing. 
To reduce vibration, it is also necessary to use special sound-absorbing structures near sources of noise or a workplace, enclose noisy units of the unit in insulating covers (gear reducers, chain, belt and other gears, impact parts, engines). When processing metal rods on automatic turret machines, the safety tube must be spring loaded or a rubber jacket inserted in the hole of this tube.

In order to reduce vibration, it is recommended to use a stiff, spring-free seat in cars, as it is a good vibration damper. Vibration acts on a person through the back, pelvis, arms. Long-term operation of the car leads to fluctuations in body parts, which negatively affects the driver. To reduce vibration, machines should be installed on a foundation deepened below the foundation of the walls, insulated from the soil by air gaps, or on specially designed shock absorbers made of steel springs or made of elastic materials.

To weaken the transmission of vibrations and noise through air ducts and pipelines, they must be connected to the fans and pumps using a flexible insert made of rubberized fabric or a rubber pipe.

It is necessary to cover vibrating surfaces and equipment with vibration absorbing and damping materials (rubber, special mastics, asbestos, bitumen, Agate plastics, etc.). At the connection points of the mating parts, shock absorbing materials (rubber, cork, cardboard, asbestos, spring shock absorbers) should be used to ensure a snug fit.

Reduce vibration in the vibration source, i.e. in the source of its formation, the following methods are possible: by excluding the impact interaction of the parts from the structure, by replacing the reciprocating movement of the parts by the rotational one, by eliminating the imbalance of rotating parts and machine components.

When working with pneumatic and electric manual machines, vibration occurs, transmitted through the handles and cases to the hands of workers, and sometimes to the legs through the medium being processed, usually when working with rammers and vibrators. To reduce vibration in this case, use handles with vibration damping or automation devices.

Personal protective equipment against vibration is used when other means are ineffective.

Shoes with shock-absorbing soles, handguards with vibration-absorbing elastic gaskets, etc. are used as personal protective equipment against vibration.

\section{Acknowledgements}

The study had no sponsorship.

\section{References}

1. A.T. Rybak, V.P. Zharov, A.V. Serdyukov, Russian Engineering Research 29(2), 194197 (2009)

2. A. Rybak, O. Lyakhnitskaya, MATEC Web of Conferences 132, 01001 (2017)

3. A.T. Rybak, A.R. Temirkanov, O.V. Lyakhnitskaya, Russian Engineering Research 38(9), $702-704$ (2018)

4. A.T. Rybak, A.R. Temirkanov, O.V. Lyakhnitskaya, Russian Engineering Research 38(3), 212-217 (2018)

5. K. Kobzev, S. Shamshura, A. Chukarin, V. Bogdanovich, V. Kasyanov, MATEC Web of Conferences 226, 01022 (2018)

6. K. Kobzev, S. Shamshura, A. Chukarin, A. Buryanovand, V. Kasyanov, MATEC Web of Conferences 226, 01023 (2018) 
7. K. Kobzev, A. Chukarin, IOP Conference Series: Earth and Environmental Science 403, 012145 (2019)

8. K.O. Kobzev, E.S. Bozhko, A.V. Mozgovoi, M.D. Molev, N.I. Stuzhenko, IOP Conference Series: Materials Science and Engineering 680, 012014 (2019)

9. K.O. Kobzev, E.S. Bozhko, A.V. Mozgovoi, E.I. Kostromina, L.G. Babenko, IOP Conference Series: Materials Science and Engineering 680, 012013 (2019)

10. N. Kobzeva, V. Terentev, I. Zolotuhina, IOP Conference Series: Materials Science and Engineering 680, 012046 (2019)

11. N. Kobzeva, V. Terentev, I. Zolotuhina, IOP Conference Series: Materials Science and Engineering 680, 012011 (2019)

12. A. Sazonova, O. Kopytenkova, E. Staseva, IOP Conference Series: Materials Science and Engineering 21, 032039 (2018) 